\title{
Comparison between Intramedullary Nailing and Percutaneous K-Wire Fixation for Fractures in the Distal Third of the Metacarpal Bone
}

\author{
Sung Jun Moon ${ }^{1}$, Jae-Won Yang ${ }^{2}$, Si Young Roh ${ }^{1}$, Dong Chul Lee ${ }^{1}$, Jin Soo Kim ${ }^{1}$ \\ ${ }^{1}$ Department of Plastic and Reconstructive Surgery, Gwangmyeong Sungae General Hospital, Gwangmyeong; ${ }^{2}$ Hand and Micro Plastic \\ Surgery, Gangnam Jaejun Plastic Clinic, Pyeongtaek, Korea
}

Background To compare clinical and radiographic outcomes between intramedullary nail fixation and percutaneous K-wire fixation for fractures in the distal third portion of the metacarpal bone.

Methods A single-institutional retrospective review identified 41 consecutive cases of metacarpal fractures between September 2009 and August 2013. Each of the cases met the inclusion criteria for closed, extra-articular fractures of the distal third of the metacarpal bone. The patients were divided by the method of fixation (intramedullary nailing or K-wire). Outcomes were compared for mean and median total active motion of the digit, radiographic parameters, and period until return to work. Complications and symptoms were determined by a questionnaire.

Results During the period under review, 41 patients met the inclusion criteria, and the fractures were managed with either intramedullary nailing $(n=19)$ or percutaneous K-wire fixation $(n=22)$. The mean and median total active range of motion and radiographic healing showed no statistically significant difference between the two groups. No union failures were observed in either group. The mean operation time was shorter by an average of 14 minutes for the percutaneous K-wire fixation group. However, the intramedullary nailing group returned to work earlier by an average of 2.3 weeks. Complications were reported only in the K-wire fixation group.

Conclusions Intramedullary nailing fixation is advisable for fractures in the distal third of the metacarpal bone. It provides early recovery of the range of motion, an earlier return to work, and lower complication rates, despite potentially requiring a wire removal procedure at the patient's request.

Keywords Metacarpal bones / Fracture fixation / Intramedullary / Fractures, closed
Correspondence: Jae-Won Yang Hand and Micro Plastic Surgery, Gangnam Jaejun Plastic Clinic, 3rd Floor, 38 Pyeongtaek 5-ro 20beon-gil, Pyeongtaek 450-020, Korea

Tel: +82-31-647-3119

Fax: +82-31-647-3119

E-mail: icebreez@hanmail.net

No potential conflict of interest relevant to this article was reported.

Received: 30 Apr 2014 • Revised: 14 Jun 2014 • Accepted: 16 Jun 2014

pISSN: 2234-6163 • elSSN: 2234-6171 • http://dx.doi.org/10.5999/aps.2014.41.6.768 • Arch Plast Surg 2014;41:768-772

\section{INTRODUCTION}

Most extra-articular fractures of the distal third metacarpal bone cause little functional impairment, and operative indications are reserved for fractures with marked shortening, complete displacement, or malrotation. Fixation techniques involve the use of K-wires, intramedullary nails, cerclage wires, plating, lag screws, tension band wires, and/or external fixators [1,2]. 
While percutaneous fixation using K-wires is a popular choice due to the simplicity of the procedure, the wires can tether to the soft tissues, particularly the sagittal bands of the extensor mechanism [3]. The possibility of pin tract infection is always present, along with a limited range of motion ( $\mathrm{ROM}$ ) during the early stage of healing up to 3-4 months after fixation.

Intramedullary nailing fixation is another method of fixation for the metacarpal fractures. The intramedullary "bouquet" osteosynthesis for fractures of the metacarpal neck was first described by Foucher [4] in 1976 and has since then gained popularity in Europe. In 2006, Orbay and Touhami [5] invented an intramedullary fixation method that enhances rotational and longitudinal stability by locking the proximal end of the metacarpal. However, for intramedullary K-wires, the main complications are 1) the migration of the K-wires, 2) the distal perforation of the head of the metacarpal, and 3) the possibility of injury of the sensitive branch of the dorsal ulnar nerve.

In this study, we aimed to compare the clinical outcomes between intramedullary nail (IMN) fixation and conventional percutaneous K-wire fixation of the extra-articular distal third metacarpal fractures.

\section{METHODS}

A single institutional retrospective review was performed for consecutive cases of displaced extra-articular distal third metacarpal fractures between September 2009 and August 2013 according to the following criteria: 1) Patients of any age with closed extra-articular distal metacarpal fractures; 2 ) no previous fractures in the same hand; 3 ) rotational deformity of more than $5^{\circ}$; 4) lateral angulation of more than $50^{\circ}$ for the small finger and $40^{\circ}$ for the index, long, and ring fingers; and 5) complete displacement of the fracture with no other associated injuries.

The review identified a total of 41 cases meeting the inclusion

\section{Fig. 1. K-wire preparation}

Dual 1.1-mm K-wires premeasured under a radiographic image intensifier were prepared for fixation. This allows the avoidance of the distal perforation of the head of the metacarpal.

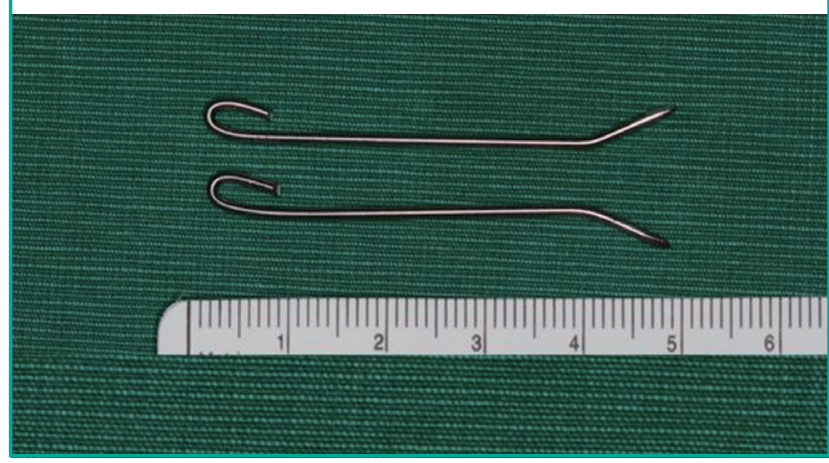

criteria. Of these, 19 were managed with IMN fixation and 22 were managed with percutaneous K-wire fixation. Patient characteristics (age, hand dominance, and gender), patterns of fracture, and preoperative radiographic parameters were collected, as well as the total active motion (TAM) of the digit and radiographic parameters (shortening, anteroposterior angulation, and number of cortices with a bridging callus), which were considered to be outcomes. A minimum of 3 cortices of the bridging callus was considered sufficient for radiographic healing. Five surgeons performed the operations. The data were analyzed using the chi-squared test and t-test. Complications and symptoms including the visual analog scale (VAS) score for pain were determined by a questionnaire.

When nailing, two 1.1-mm K-wires were used for each IMN fixation, and two K-wires of either $0.9 \mathrm{~mm}$ or $1.1 \mathrm{~mm}$ were used for percutaneous fixations.

Prior to the preparation of the IMN, a reduction of the metacarpal bone was performed by using the Jahss maneuver. Under $\mathrm{C}$-arm control, an adequate length of the K-wire was measured in advance, and the distal part of the K-wire was bent by about $30^{\circ}$ while the proximal end was bent more than $90^{\circ}$ for locking (Fig. 1).

In case of the fifth metacarpal bone, the soft tissue and skin were pulled tightly to the radial side and a small incision was made on the ulnar side of the fifth metacarpal bone base level in order to avoid the anatomical location of the dorsal branch of the ulnar nerve (Fig. 2) [6-8]. Under the direct visualization of the notch of the cortex, the metaphyseal cortex was perforated with an awl, and the nails were advanced using a small mallet in order to prevent the supplementary bending of the nails during

\section{Fig. 2. Avoidance of ulnar nerve branch}

The soft tissue and skin were pulled tightly to the radial side and a small incision was made on the ulnar side of the fifth metacarpal bone in order to avoid the location of the dorsal sensory branch of the ulnar nerve (red arrow).

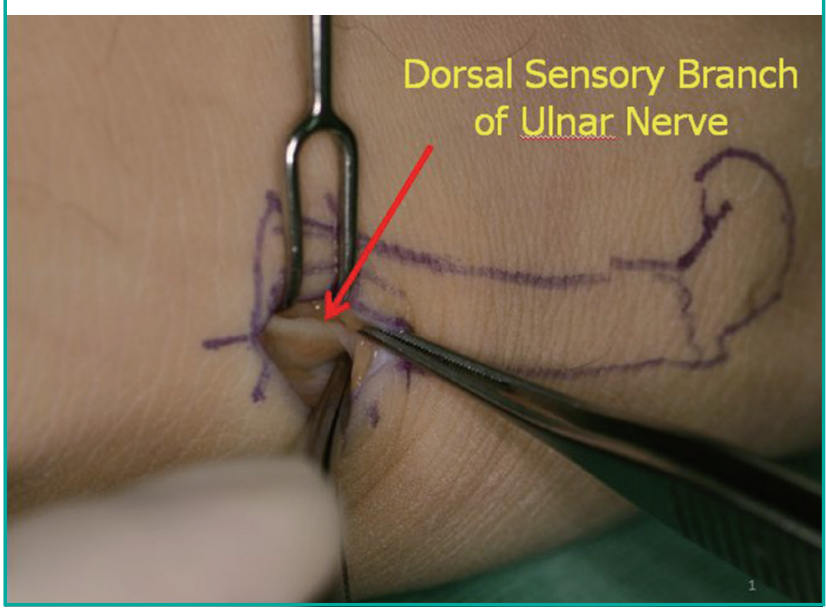




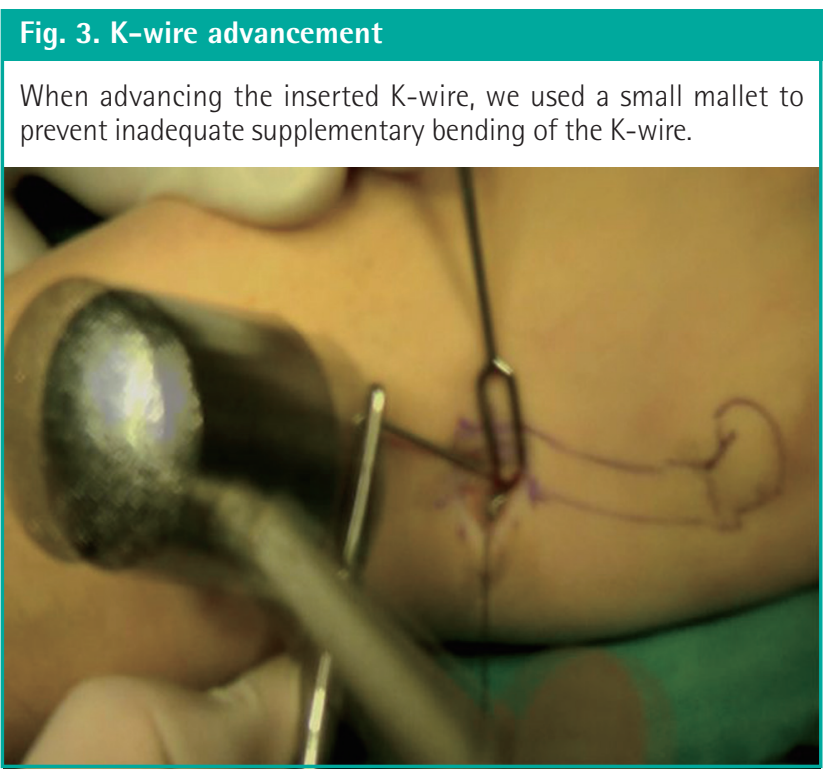

Fig. 4. Stability enhancement

Each bent K-wire distal tip was inserted in the opposite direction in order to increase stability.

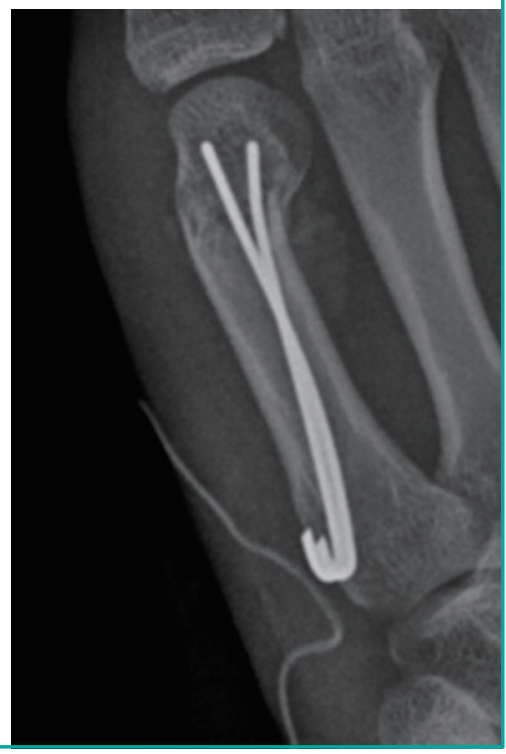

insertion (Fig. 3). The bent distal portions of the nails were placed at an angle to each other in order to resist the torsion forces (Fig. 4). The proximal "J-shaped hook" portions were fitted to the cortex (Fig. 5) [9].

The reduced metacarpal bones were protected with in a wellmolded dorsal short-arm splint in the intrinsic-plus position, which maintained the wrist at an extension of approximately $30^{\circ}$, the metacarpophalangeal joint flexed at approximately $70^{\circ}$, and the interphalangeal joints fully extended [10], and which was removed 2 weeks after surgery, after which patients were allowed to perform day-to-day activities as tolerated. Early physical therapy was performed for all patients between 3 and 5 postoperative weeks. Surgical outcomes were compared between

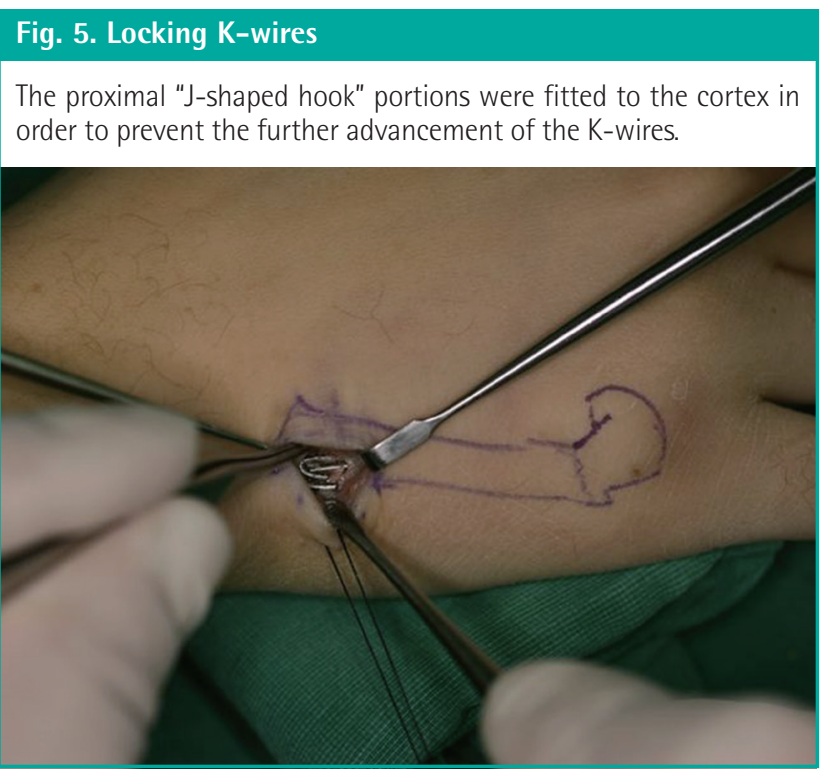

Table 1. Patient demographics and mean follow-up period

\begin{tabular}{|lcc|}
\hline Characteristic & $\begin{array}{c}\text { Intramedullary } \\
\text { nailing }(\mathbf{n}=\mathbf{1 9})\end{array}$ & $\begin{array}{c}\text { Percutaneous } \\
\text { K-wire }(\mathbf{n = 2 2})\end{array}$ \\
\hline Mean age (yr) & $28(15-49)$ & $34(19-71)$ \\
Fractured site dominant $(\mathrm{n})$ & 17 & 17 \\
Gender (male/female) & $14 / 5$ & $19 / 3$ \\
Injury to surgery (day) & $4(1-16)$ & $3(1-15)$ \\
Mean follow-up (wk) & 11 & 15 \\
\hline
\end{tabular}

\section{Table 2. Fracture locations}

\begin{tabular}{|lcc|}
\hline Characteristic & $\begin{array}{c}\text { Intramedullary } \\
\text { nailing }(\mathbf{n = 1 9 )}\end{array}$ & $\begin{array}{c}\text { Percutaneous } \\
\text { K-wire }(\mathbf{n = 2 2 )}\end{array}$ \\
\hline 2nd metacarpal bone $(n)$ & 0 & 1 \\
3rd metacarpal bone $(n)$ & 2 & 2 \\
4th metacarpal bone $(n)$ & 3 & 2 \\
5th metacarpal bone $(n)$ & 14 & 17 \\
\hline
\end{tabular}

the two methods of fixation. Data were analyzed using the chisquared test and the t-test. A P-value of $<0.05$ was considered significant. Statistical analysis was performed using statistical software (SPSS ver. 20.0, IBM Corp., Armonk, NY, USA).

\section{RESULTS}

Patient demographics such as age and male-to-female ratio did not significantly differ between the two groups (Tables 1, 2).

In line with the retrospective inclusion criteria, the mean preoperative angulation and shortening were clinically significant in both groups. Both methods were effective in correcting angulation and shortening, with the postoperative angulation of $4.2^{\circ}$ and $5.6^{\circ}$ and postoperative shortening of $0.9 \mathrm{~mm}$ and $0.5 \mathrm{~mm}$ in 


\section{Table 3. Results}

\begin{tabular}{|c|c|c|c|}
\hline Characteristic & $\begin{array}{l}\text { Intramedullary } \\
\text { nailing }(n=19)\end{array}$ & $\begin{array}{l}\text { Percutaneous } \\
\text { K-wire }(n=22)\end{array}$ & P-value \\
\hline Duration of surgery (min) & 48 & 34 & \\
\hline Preoperative angulation $\left({ }^{\circ}\right)$ & $48(20-74)$ & $41(0-82)$ & Not significant \\
\hline Postoperative angulation $\left({ }^{\circ}\right)$ & $4.2(0-6)$ & $5.6(0-8)$ & Not significant \\
\hline Preoperative shortening (mm) & $8.1(0-19)$ & $7.7(0-17)$ & Not significant \\
\hline Postoperative shortening (mm) & $0.9(0-4)$ & $0.5(0-3)$ & Not significant \\
\hline Radiographic healing (wk) & 5.4 & 5.2 & Not significant \\
\hline Loss of reduction (n) & 0 & 0 & Not significant \\
\hline Early TAM of the digit $\left({ }^{\circ}\right)$ & 225 & 135 & $<0.001$ \\
\hline Late TAM of the digit $\left({ }^{\circ}\right)$ & 250 & 245 & Not significant \\
\hline Period to return to work (wk) & 5.5 & 7.8 & 0.024 \\
\hline
\end{tabular}

the IMN and K-wire groups. Likewise, a radiographic bone union was observed at around 5 weeks for both the groups.

TAM was significantly different between the groups at 5 weeks ( $225^{\circ}$ in the IMN group and $135^{\circ}$ in the K-wire group), but this difference was not observed at 10 weeks postoperatively. In addition, patients who underwent IMN fixation returned to work earlier (5.5 weeks in the IMN group and 7.8 weeks in the Kwire group) (Table 3 ).

Percutaneous K-wire fixation was associated with three superficial wound infections $(3 / 22)$, which were adequately treated with an intravenous (IV) antibiotic regimen and twice daily dressing changes. There were no such complications in the IMN group. According to the questionnaire survey conducted at 2 weeks postoperation, discomfort $(\mathrm{n}=11)$, pain (mean VAS Score, 21/100), and swelling of the hand dorsum $(n=9)$ were reported by the K-wire group. Patients in the IMN group reported no discomfort or swelling and less pain (mean VAS score, 9/100) (Table 4).

\section{DISCUSSION}

Among various closed metacarpal fractures, transverse and short oblique fractures of the neck are very common. Not all fractures require operative intervention, but significant bone shortening and angulation are associated with poor long-term functional outcomes. Plates and cerclage wires may more than adequately address these relatively simple fractures; however, the increase in the operative time and soft tissue dissection is difficult to justify when considering the availability of percutaneous methods using K-wires or IMN.

Previously, these two percutaneous methods were compared between a non-randomized clinical trial and a retrospective review $[3,10]$. In the study by Wong et al. [3], single IMN fixation was compared to transverse K-wire fixation. The measured outcomes were radiological union and postoperative pain, move-
Table 4. Complications

\begin{tabular}{|lcc|}
\hline Characteristic & $\begin{array}{c}\text { Intramedullary } \\
\text { nailing }(\mathbf{n = 1 9 )}\end{array}$ & $\begin{array}{c}\text { Percutaneous } \\
\text { K-wire }(\mathbf{n = 2 2 )}\end{array}$ \\
\hline Pin site infection (n) & 0 & 3 \\
Mean visual analog scale score & 9 & 21 \\
Discomfort by K-wire exposure $(\mathrm{n})$ & 0 & 11 \\
Hand dorsum swelling $(\mathrm{n})$ & 0 & 9 \\
\hline
\end{tabular}

ment, and grip strength, none of which were found to be significantly different between the groups. In the retrospective study by Schadel-Hopfner et al. [10], the comparison was between multiple crossed retrograde percutaneous K-wires and single anterograde IMN. Of the outcomes evaluated, the metacarpophalangeal joint ROM, pain, and Steel scores were significantly in favor of IMN.

Our own retrospective review revealed that IMN were associated with subtle improvements in the postoperative course. While preoperative angulation and shortening had improved in both groups, patients reported satisfaction regarding comfort and tenderness overwhelmingly in the favor of the intramedullary method. Additionally, patients who received IMN fixation returned to work significantly earlier by a mean difference of 16 days.

Although a significant difference in the early TAM was observed at the fifth operative week (Table 3 ), no statistically significant difference was observed according to the TAM of digits at the 10th operative week. For the percutaneous K-wire insertion group, the extension deficit disappeared completely at approximately 1 week or 2 weeks after wire removal and there were no cases of permanent extensor problems. Therefore, we concluded that the extension lag was just caused by the tethering effect of wires and extensor irritation was not serious enough to cause permanent problems.

The most significant study limitation is the retrospective nature of its design. The assumption was that patients and metacarpal fractures were relatively homogeneous irrespective of the fixation method and that the fixation type was based purely on surgeon preference. However accurate this assumption may be, the study results should be interpreted with a potential selection bias in mind. It is also possible that the lack of external hardware may have encouraged a more active participation among patients who had undergone IMN fixation.

In conclusion, our retrospective review has shown that both IMN and percutaneous K-wire fixations were able to appropriately reduce the indicated metacarpal distal third fractures. Outcomes of the 10th postoperative week were excellent for both in terms of TAM and union rates. Clinically significant differences 
were observed in the favor of IMN fixations, for early TAM and the time required to return to work.

\section{REFERENCES}

1. Black D, Mann RJ, Constine R, et al. Comparison of internal fixation techniques in metacarpal fractures.J Hand Surg Am 1985; 10:466-72.

2. Kawamura K, Chung KC. Fixation choices for closed simple unstable oblique phalangeal and metacarpal fractures. Hand Clin 2006;22:287-95.

3. Wong TC, Ip FK, Yeung SH. Comparison between percutaneous transverse fixation and intramedullary $\mathrm{K}$-wires in treating closed fractures of the metacarpal neck of the little finger.J Hand Surg Br 2006;31:61-5.

4. Foucher G. "Bouquet" osteosynthesis in metacarpal neck fractures: a series of 66 patients. J Hand Surg Am 1995;20: S86-90.

5. Orbay JL, Touhami A. The treatment of unstable metacarpal and phalangeal shaft fractures with flexible nonlocking and locking intramedullary nails. Hand Clin 2006;22:27986.

6. Botte MJ, Cohen MS, Lavernia CJ, et al. The dorsal branch of the ulnar nerve: an anatomic study.J Hand Surg Am 1990; 15:603-7.

7. Itadera E, Hiwatari R, Moriya $\mathrm{H}$, et al. Closed intramedullary fixation for metacarpal fractures using J-shaped nail. Hand Surg 2008; 13:139-45.

8. Polat $\mathrm{O}$, Comert A, Atalar H, et al. Safe percutaneous pinning for subcapital fifth metacarapl fractures: an anatomical stusy. Acta Orthop Bras 2011;19:106-9.

9. Rhee SH, Lee SK, Lee SL, et al. Prospective multicenter trial of modified retrograde percutaneous intramedullary Kirschner wire fixation for displaced metacarpal neck and shaft fractures. Plast Reconstr Surg 2012;129:694-703.

10. Schadel-Hopfner M, Wild M, Windolf J, et al. Antegrade intramedullary splinting or percutaneous retrograde crossed pinning for displaced neck fractures of the fifth metacarpal? Arch Orthop Trauma Surg 2007; 127:435-40. 